\title{
Molecular Targets and Mechanisms of Scutellariae radix-Coptidis rhizoma Drug Pair for the Treatment of Ulcerative Colitis Based on Network Pharmacology and Molecular Docking
}

\author{
Kai Niu, ${ }^{1}$ Qifang Li $\mathbb{D}^{2},{ }^{2}$ Yuan Liu $\mathbb{D}^{1},{ }^{1}$ Yi Qiao, ${ }^{3}$ Bingbing Li $\mathbb{D}^{1},{ }^{1}$ Chao Wei, ${ }^{1}$ Kunrui Wang, \\ Lu'an Cui, ${ }^{1}$ Canlei Zheng, ${ }^{1}$ Rong Wang, ${ }^{1}$ Li Zhang, ${ }^{1}$ Honghua Zhang, ${ }^{4}$ Bing Sun $\mathbb{D}^{1},{ }^{1}$ \\ and Bin Yu $\mathbb{D i D}^{1}$ \\ ${ }^{1}$ College of Integrated Chinese and Western Medicine, Jining Medical University, Jining 272067, China \\ ${ }^{2}$ Department of Traditional Chinese Medicine, Affiliated Hospital of Jining Medical University, Jining 272060, China \\ ${ }^{3}$ School of Public Health, Jining Medical University, Jining 272067, China \\ ${ }^{4}$ College of Pharmaceutical Sciences, Hangzhou Normal University, Hangzhou 311121, China
}

Correspondence should be addressed to Bin Yu; yubinsd@yeah.net

Received 2 April 2021; Accepted 28 May 2021; Published 4 June 2021

Academic Editor: Dejan Stojkovic

Copyright ( 92021 Kai Niu et al. This is an open access article distributed under the Creative Commons Attribution License, which permits unrestricted use, distribution, and reproduction in any medium, provided the original work is properly cited.

This study aims to analyze the targets of the effective active ingredients of Scutellariae radix-Coptidis rhizoma drug pair (SCDP) in ulcerative colitis (UC) by network pharmacology and molecular docking and to explore the associated therapeutic mechanism. The effective active ingredients and targets of SCDP were determined from the TCMSP database, and the drug ingredient-target network was constructed using the Cytoscape software. The disease targets related to UC were searched in GeneCards, DisGeNET, OMIM, and DrugBank databases. Then, the drug ingredient and disease targets were intersected to construct a protein-protein interaction network through the STRING database. The Metascape database was used for the Gene Ontology and Kyoto Encyclopedia of Genes and Genomes pathway enrichment analyses of the predicted targets of SCDP for UC. The Autodock software was used for molecular docking between the main active ingredient and the core target to evaluate the binding ability. SCDP has 43 effective active ingredients and 134 intersection targets. Core targets included AKT1, TP53, IL-6, VEGFA, CASP3, JUN, TNF, MYC, EGFR, and PTGS2. GO functional enrichment analysis showed that biological process was mainly associated with a cytokine-mediated signaling pathway, response to an inorganic substance, response to a toxic substance, response to lipopolysaccharide, reactive oxygen species metabolic process, positive regulation of cell death, apoptotic signaling pathway, and response to wounding. KEGG enrichment analysis showed main pathway concentrations were related to pathways in cancer, AGE-RAGE signaling pathway in diabetic complications, bladder cancer, IL-17 signaling pathway, apoptosis, p53 signaling pathway, and PI3K-Akt signaling pathway. The drug active ingredient-core target-key pathway network contains 41 nodes and 108 edges, of which quercetin, wogonin, baicalein, acacetin, oroxylin A, and beta-sitosterol are important active ingredients; PTGS2, CASP3, TP53, IL-6, TNF, and AKT1 are important targets; and the pathways involved in UC treatment include pathways in cancer, PI3K-Akt signaling pathway, AGE-RAGE signaling pathway in diabetic, apoptosis, IL-17 signaling pathway and herpes simplex infection. The active ingredient has a good binding capacity to the core target. SCDP key active ingredients are mainly quercetin, wogonin, baicalein, acacetin, oroxylin A, and beta-sitosterol, which function mainly by regulating targets, such as PTGS2, CASP3, TP53, IL-6, TNF, and AKT1, and are associated with multiple signaling pathways as pathways in cancer, PI3K-Akt signaling pathway, apoptosis, IL-17 signaling pathways. 


\section{Introduction}

Ulcerative colitis (UC), a type of inflammatory bowel disease (IBD), is a chronic inflammatory disease of the intestine characterized by abnormal intestinal mucosal structure, changes in intestinal bacterial composition, and systemic biochemical dysfunction. It is an incurable disease with low mortality [1]. The incidence of this disease is 11.60 per 100,000 individuals and 24.5 per 100,000 individuals in China and Hong Kong, respectively, which is lower than the incidence in Western countries but shows an increasing annual trend [2-5]. The main clinical symptoms are abdominal pain, diarrhea, bloody purulent stools, and weight loss accompanied by numerous neutrophils, macrophages, and inflammatory factors infiltrating the intestinal mucosa. The pathogenesis of this disease is not clear, and studies suggest a complex interaction among host genetic factors, immunity, and microbial environmental exposure [6]. In addition to aminosalicylic acid preparations and corticosteroids, immunomodulators, Janus kinase inhibitors, and some biological agents, such as tumor necrosis factor-alpha (TNF- $\alpha$ ), interleukin (IL)-17A, and IL-12/IL-23p40 antibodies, have been applied in the clinical treatment of UC [7]. However, more and more patients eventually become refractory or intolerant to the side effects or complications of drugs, and the persistence of chronic inflammatory conditions often becomes a risk factor for inducing colorectal cancer $[8,9]$. Under the circumstance, traditional Chinese medicines (TCMs) play an important role in the treatment of UC and are regarded as complementary and alternative medicine treatment options. Also, much evidence has shown that TCM therapies, including Chinese herbal medicine, Chinese patent medicine, acupuncture, moxibustion, have potentially positive effects on UC $[10,11]$.

Scutellariae radix-Coptidis rhizoma drug pair is the main ingredient herb for clearing away heat and dampness. Their compatibility is often used to treat damp-heat syndromes, dysentery, diarrhea, and other diseases [12-14]. Furthermore, they are the main ingredient drugs in the classical prescriptions as Gegen Qinlian decoction, Wumei pill, Banxia Xiexin decoction, and Shaoyao decoction, and others [15-18]. The combination of Scutellaria baicalensis and Coptis chinensis is mainly responsible for clearing away heat and dampness and has an antidiarrheal and antidysentery efficacy. Furthermore, $\mathrm{Hu}$ et al. used a database to analyze the medication regularity of TCM compounds in the treatment of UC. Results showed that Scutellaria baicalensis and Coptis chinensis were among the top 26 drugs with the highest medication, of which and Coptis chinensis was the most frequently used drug [19]. Professor Jingri Xie advocated that Scutellaria baicalensis, Coptis chinensis, Phellodendron amurense, and Sophora flavescens can be used together and they are particularly suitable for patients with the damp-heat syndrome, such as abdominal bloat, bitter mouth, nausea, vomiting, and/or yellow tongue coating [20]. Previous work in our laboratory also confirmed that Scutellaria baicalensis and Coptis chinensis are the drugs which were used more frequently in the treatment of UC and are commonly paired drugs to treat damp-heat intrinsic syndrome [21, 22].
One proposed mechanism of the Scutellariae radixCoptidis rhizoma drug pair (SCDP) in the treatment of UC is that baicalein can reduce the number of apoptotic intestinal epithelial cells due to its ability to reduce the expression of the 78 -kDa glucose-regulated protein (GRP78) and caspase3 [23]. Researchers have found that berberine-nanostructured lipid carriers can block the nuclear translocation of nuclear factor-kappa B (NF- $\kappa \mathrm{B})$, reduce the expression of the proinflammatory cytokines IL- $1 \beta$, IL-6, matrix metalloproteinase-9, chemokine motif receptor 1 (CX3CR1), cyclooxygenase-2 (COX-2), and telomerase reverse transcriptase, and increase the expression of the tight junction scaffolding protein zonula occludens-1, to play a therapeutic role in UC [24]. Gegen Qinlian decoction, comprising herbs Scutellariae radix and Coptidis rhizoma, exert therapeutic effects by inhibiting the expression of toll-like receptor 4 (TLR4)/NF- $\kappa \mathrm{B}$ pathway and suppressing the inflammatory factors secretion as TNF- $\alpha$, IL- 6 , IL- $1 \beta$, and IL- 4 in the colon of UC animal models [15]. In addition, a recent report has revealed that baicalin, which was the main ingredient of Coptidis rhizoma, can exert a therapeutic effect on UC through increasing glutathione peroxidase and superoxide dismutase contents and inhibiting oxidative stress response as well as apoptosis [10].

Network pharmacology and molecular docking are new technologies based on systems biology and databased molecular correlation analysis in the exploration of new drugs and prediction of drug targets. Network pharmacology emphasizes multi-ingredient-multitargets-multipathway regulation of the signal pathway. Many studies have confirmed that network pharmacology is significant for determining the target of drugs and screening the active ingredients of drugs [25-27]. In this study, the mechanism of Scutellariae radix-Coptidis rhizoma drug pair in the treatment of UC was analyzed by network pharmacology technology and molecular docking approaches to help better guide clinical treatment and mechanism research.

\section{Materials and Methods}

\subsection{SCDP Ingredients Collection and Target Gene Prediction.} The Traditional Chinese Medicine Systems Pharmacology (TCMSP) database and analysis platform (https://tcmspw. com/tcmsp.php) was used for the active ingredient screening. Oral bioavailability $(\mathrm{OB}) \geq 30 \%$ and drug-likeness (DL) $\geq 0.18$ were used as absorption, distribution, metabolism, and excretion parameters. Target genes were predicted using TCMSP and the Swiss Target Prediction database (http:// www.swisstargetprediction.ch/) after identifying the active ingredients. The names of the predicted target genes were standardized from the UniProt database (https://www. uniprot.org/). The SCDP ingredients and target gene network were drawn using the Cytoscape software, and the top 10 active ingredients were identified by their degree values.

\subsection{Prediction of UC-Related Pathogenic Genes.} GeneCards (https://www.genecards.org/), DisGeNET (https://www.disgenet.org/), OMIM (http://www.omim. org/), and DrugBank (https://www.drugbank.ca/) were 
selected to obtain UC-related pathogenic genes. Both databases can be used to find the most cutting-edge diseaserelated genes. All the databases were used "ulcerative colitis" as the keyword.

\subsection{Protein-Protein Interaction (PPI) Analysis and Hub} Genes. PPI is particularly important for biological process analyses and is vital for understanding complex mechanisms in a living cell. PPI network mapping was performed on the obtained bioactive ingredients and disease targets using the Search Tool for the Retrieval of Interacting Genes (STRING) database (http://string-db.org/) with the species limited to "Homo sapiens" and a confidence score of $>0.4$. Next, the interaction files were downloaded and imported into Cytoscape to visualize the PPI network and analyze the topological value. Then, based on the MCODE plug-in, the targets with a median value and a median value that was not less than the average value were recognized as the key targets of SCDP for the treatment of UC. And the top 10 ranked proteins were defined as hub targets based on the degree level.

2.4. Enrichment Analysis of Gene Ontology (GO) and Kyoto Encyclopedia of Genes and Genomes (KEGG). The predicted targets were imported into the Metascape database (http:// metascape.org/) for GO and KEGG enrichment analyses by entering the list of intersection target gene names and selecting the species as "Homo sapiens" for customized analysis with a cutoff $P$ value of $<0.01$. According to the results, a network visualization was constructed by the tools in the bioinformatics website (http://www.bioinformatics. com.cn).

\subsection{SCDP Drug Ingredient-Target Genes-Pathway Interaction} Network. The SCDP active ingredients predicted target genes, and the top 20 key pathways for enrichment analysis were imported into the Cytoscape software to construct the drug ingredient-target genes-pathway interaction network. Topological analysis was performed on the constructed network according to the values of degree centrality, betweenness centrality, and closeness centrality.

2.6. Molecular Docking. Molecular docking is used to interpret the binding area of small molecule ligands and macromolecular receptors through computer simulation and then calculates the physical and chemical parameters for predicting the affinity between the two. Receptor and ligand files were downloaded from PubChem (https://pubchem. ncbi.nlm.nih.gov/) and PDB (https://www.rcsb.org) databases.

And then, molecular docking was performed using the AutoDockTools software. The docking range between the protein and the active ingredient was set using AutoGrid, and molecular docking was performed by running the "Genetic Algorithm" using Docking settings, with binding energy less than 0 indicating a good potential for ligand binding to the receptor.

\section{Results}

3.1. SCDP Ingredients Collection and Target Gene Prediction. According to the two screening conditions of $\mathrm{OB}$ value and DL index, 50 active ingredients of SCDP, including 36 ingredients in Scutellariae radix and 14 ingredients in Coptidis rhizoma, were obtained from TCMSP. Eight ingredients were eliminated because they showed no predicted targets in either the TCMSP or Swiss Target Prediction databases. Finally, 43 ingredients, including 32 in Scutellariae radix, 11 in Coptidis rhizoma, and coptisine and epiberberine as two common ingredients, were obtained (Table 1). Then the target genes of 43 active ingredients were collected for target genes prediction in TCMSP and Swiss Target Prediction. After merging the data and deleting duplicates, 223 target genes were finally distinguished.

\subsection{Drug Active Ingredient-Target Gene Interaction Network.} Drug active ingredient-target gene interaction networks were obtained by importing the active ingredients and predicted genes into Cytoscape 3.8.0. There are 266 nodes and 824 edges (Figure 1). The edges represent the interrelationships between the drug and active ingredients and between the ingredients and targets. Meanwhile, the degree value represents the number of routers connected to each node in the network. The higher the degree value, the more likely the node plays a key role in the network. In this network, the same compound acted on multiple targets. Simultaneously, multiple compounds shared the same target. Furthermore, the top 10 key active ingredients and 19 core targets (degree $\geq 10$ ) were screened according to the degree value as shown in Tables 2 and 3.

The purple squares represent the drug Scutellariae radix and Coptidis rhizoma. The green octagons represent the ingredients in Scutellariae radix, while green hexagons represent the ingredients of Coptidis rhizoma. The red circle represents the common active ingredient of SCDP. The blue diamond represents the predicted target. The area and color transparency of the node represent its value. The darker the area and color, the more important the node. SCDP: Scutellariae radix-Coptidis rhizoma drug pair.

3.3. Prediction of UC-Related Pathogenic Genes. The disease genes related to UC were collected from GeneCards, DisGeNET, OMIM, and DrugBank, with "ulcerative colitis" as the keyword. Taking 1.795 and 4.51 as the median "Relevance score," 1111 relevant targets were screened in GeneCards. Meanwhile, 1458, 552, and 65 pathogenic genes were obtained from the DisGeNET, OMIM, and DrugBank databases, respectively. The UniProt database was used to standardize target names, and finally a total of 2386 UCrelated pathogenic target genes were obtained after collating and removing duplicates.

3.4. Construction of the PPI Network and Core Target Screening. A total of 134 intersection targets were obtained by screening drug ingredient targets and disease targets 
Table 1: Ingredients in Scutellariae radix-Coptidis rhizoma drug pair.

\begin{tabular}{|c|c|c|c|c|}
\hline Drug source & Molecule ID & Molecule name & $\mathrm{OB}(\%)$ & $\mathrm{DL}$ \\
\hline \multirow{32}{*}{ Scutellariae radix } & MOL001689 & Acacetin & 34.97 & 0.24 \\
\hline & MOL000173 & Wogonin & 30.68 & 0.23 \\
\hline & MOL000228 & (2R)-7-Hydroxy-5-methoxy-2-phenylchroman-4-one & 55.23 & 0.2 \\
\hline & MOL002714 & Baicalein & 33.52 & 0.21 \\
\hline & MOL002909 & 5,7,2,5-Tetrahydroxy-8,6-dimethoxyflavone & 33.82 & 0.45 \\
\hline & MOL002910 & Carthamidin & 41.15 & 0.24 \\
\hline & MOL002913 & Dihydrobaicalin_qt & 40.04 & 0.21 \\
\hline & MOL002914 & Eriodyctiol (flavanone) & 41.35 & 0.24 \\
\hline & MOL002915 & Salvigenin & 49.07 & 0.33 \\
\hline & MOL002917 & $5,2^{\prime}, 6^{\prime}$-Trihydroxy-7,8-dimethoxyflavone & 45.05 & 0.33 \\
\hline & MOL002925 & $5,7,2^{\prime}, 6^{\prime}$-Tetrahydroxyflavone & 37.01 & 0.24 \\
\hline & MOL002927 & Skullcapflavone II & 69.51 & 0.44 \\
\hline & MOL002928 & Oroxylin A & 41.37 & 0.23 \\
\hline & MOL002932 & Panicolin & 76.26 & 0.29 \\
\hline & MOL002933 & $5,7,4^{\prime}$-Trihydroxy-8-methoxyflavone & 36.56 & 0.27 \\
\hline & MOL002934 & Neobaicalein & 104.34 & 0.44 \\
\hline & MOL002937 & Dihydrooroxylin & 66.06 & 0.23 \\
\hline & MOL000358 & Beta-sitosterol & 36.91 & 0.75 \\
\hline & MOL000359 & Sitosterol & 36.91 & 0.75 \\
\hline & MOL000525 & Norwogonin & 39.4 & 0.21 \\
\hline & MOL000552 & $5,2^{\prime}$-Dihydroxy-6,7,8-trimethoxyflavone & 31.71 & 0.35 \\
\hline & MOL000073 & Ent-epicatechin & 48.96 & 0.24 \\
\hline & MOL000449 & Stigmasterol & 43.83 & 0.76 \\
\hline & MOL001458 & Coptisine & 30.67 & 0.86 \\
\hline & MOL001490 & $\operatorname{Bis}[(2 S)$-2-ethylhexyl] benzene-1,2-dicarboxylate & 43.59 & 0.35 \\
\hline & MOL002879 & Diop & 43.59 & 0.39 \\
\hline & MOL002897 & Epiberberine & 43.09 & 0.78 \\
\hline & MOL008206 & Moslosooflavone & 44.09 & 0.25 \\
\hline & MOL010415 & 11,13-Eicosadienoic acid, methyl ester & 39.28 & 0.23 \\
\hline & MOL012245 & 5,7,4'-Trihydroxy-6-methoxyflavanone & 36.63 & 0.27 \\
\hline & MOL012246 & $5,7,4^{\prime}$-Trihydroxy-8-methoxyflavanone & 74.24 & 0.26 \\
\hline & MOL012266 & Rivularin & 37.94 & 0.37 \\
\hline \multirow{11}{*}{ Coptidis rhizoma } & MOL001454 & Berberine & 36.86 & 0.78 \\
\hline & MOL002894 & Berberrubine & 35.74 & 0.73 \\
\hline & MOL002897 & Epiberberine & 43.09 & 0.78 \\
\hline & MOL002903 & $(R)$-Canadine & 55.37 & 0.77 \\
\hline & MOL002904 & Berlambine & 36.68 & 0.82 \\
\hline & MOL002907 & Corchoroside A_qt & 104.95 & 0.78 \\
\hline & MOL000622 & Magnograndiolide & 63.71 & 0.19 \\
\hline & MOL000785 & Palmatine & 64.6 & 0.65 \\
\hline & MOL000098 & Quercetin & 46.43 & 0.28 \\
\hline & MOL001458 & Coptisine & 30.67 & 0.86 \\
\hline & MOL002668 & Worenine & 45.83 & 0.87 \\
\hline
\end{tabular}

OB: oral bioavailability; DL: drug-likeness.

using the Wayne diagram. The PPI network was construed by the STRING databases of the 134 targets, as shown in Figure 2, with 134 nodes, 2612 edges, an average node degree of 39 , and a PPI enrichment $p<1.0 e-16$.

The obtained TSV format files of the PPI network were imported into the Cytoscape software for visualization analysis. As the interactions between proteins in the PPI networks are reciprocal, it is usually classified as an undirected graph. Some regions with high density were present in the PPI complex network, and they are called a community or module. The networks within modules are potential subnetworks of the PPI network, with a high density of connections in the subnetworks and few connections in other regions. Therefore, modules are considered to be biologically significant sets. The sets have two meanings: (1) protein complex, (i.e., multiple proteins combine to form a complex, which then plays a biological role); (2) functional module (e.g., proteins located in the same pathway interact more closely). The MCODE plug-in was then applied to analyze the interactions using a molecular complex detection algorithm, and 3 module clusters were obtained (Figure 3). According to the degree values, the top 10 core targets were identified as AKT1, TP53, IL-6, VEGFA, CASP3, JUN, TNF, MYC, EGFR, and PTGS2 (Figure 3), whose full names are provided in Table 4. 


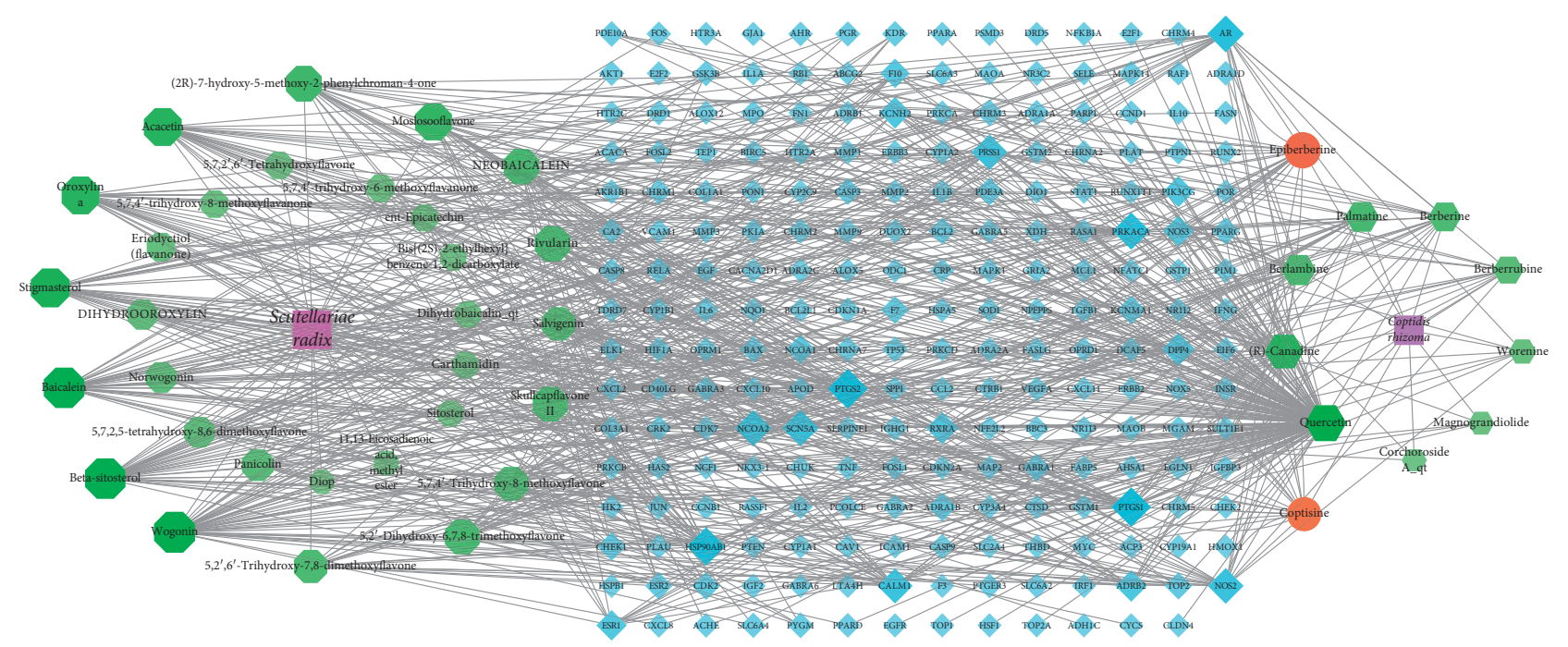

FIGURE 1: Drug ingredient-target interaction network. The purple squares represent the drug Scutellariae radix and Coptidis rhizoma. The green octagons represent the ingredients in Scutellariae radix, while green hexagons represent the ingredients of Coptidis rhizoma. The red circle represents the common active ingredient of SCDP. The blue diamond represents the predicted target. The area and color transparency of the node represent its value. The darker the area and color, the more important the node. SCDP, Scutellariae radix-Coptidis rhizoma drug pair.

TABle 2: Top 10 key active ingredients in Scutellariae radix-Coptidis rhizoma drug pair.

\begin{tabular}{lllr}
\hline Rank & Molecule ID & Name & Degree $\geq 24$ \\
\hline 1 & MOL000098 & Quercetin & 151 \\
2 & MOL000173 & Wogonin & 46 \\
3 & MOL000358 & Beta-sitosterol & 38 \\
4 & MOL002714 & Baicalein & 38 \\
5 & MOL000449 & Stigmasterol & 32 \\
6 & MOL002903 & (R)-Canadine & 32 \\
6 & MOL002928 & Oroxylin A & 27 \\
8 & MOL001689 & Acacetin & 27 \\
9 & MOL008206 & Moslosooflavone & 25 \\
10 & MOL002897 & Epiberberine & 24 \\
\hline
\end{tabular}

TABlE 3: Core targets of active ingredients in the Scutellariae radix-Coptidis rhizoma drug pair.

\begin{tabular}{lcccc}
\hline Rank & Name & Degree $\geq 10$ & Closeness centrality & Neighbourhood connectivity \\
\hline 1 & PTGS2 & 37 & 0.5248 & 22.3429 \\
2 & PTGS1 & 33 & 0.5166 & 22.9375 \\
3 & HSP90AB1 & 30 & 0.5009 & 23.4667 \\
4 & PRKACA & 26 & 0.5009 & 24.5200 \\
5 & AR & 25 & 0.4514 & 25.5652 \\
6 & SCN5A & 24 & 0.4775 & 26.5652 \\
7 & NCOA2 & 24 & 0.4809 & 24.7391 \\
8 & PRSS1 & 23 & 0.4484 & 27.5238 \\
9 & NOS2 & 23 & 0.3228 & 19.6190 \\
10 & CALM1 & 22 & 0.3529 & 21.5909 \\
11 & DPP4 & 17 & 0.4368 & 29.4706 \\
12 & RXRA & 16 & 0.4530 & 31.4667 \\
13 & ADRB2 & 0.4625 & 32.8000 \\
14 & KCNH2 & 15 & 0.4366 & 30.9231 \\
15 & PIK3CG & 15 & 0.4515 & 32.4286 \\
16 & NOS3 & 14 & 0.3973 & 29.8182 \\
17 & ESR1 & 14 & 0.3078 & 18.1818 \\
18 & F10 & 13 & 0.4213 & 32.2727 \\
19 & NCOA1 & 11 & 0.3236 & 24.0000 \\
\hline
\end{tabular}




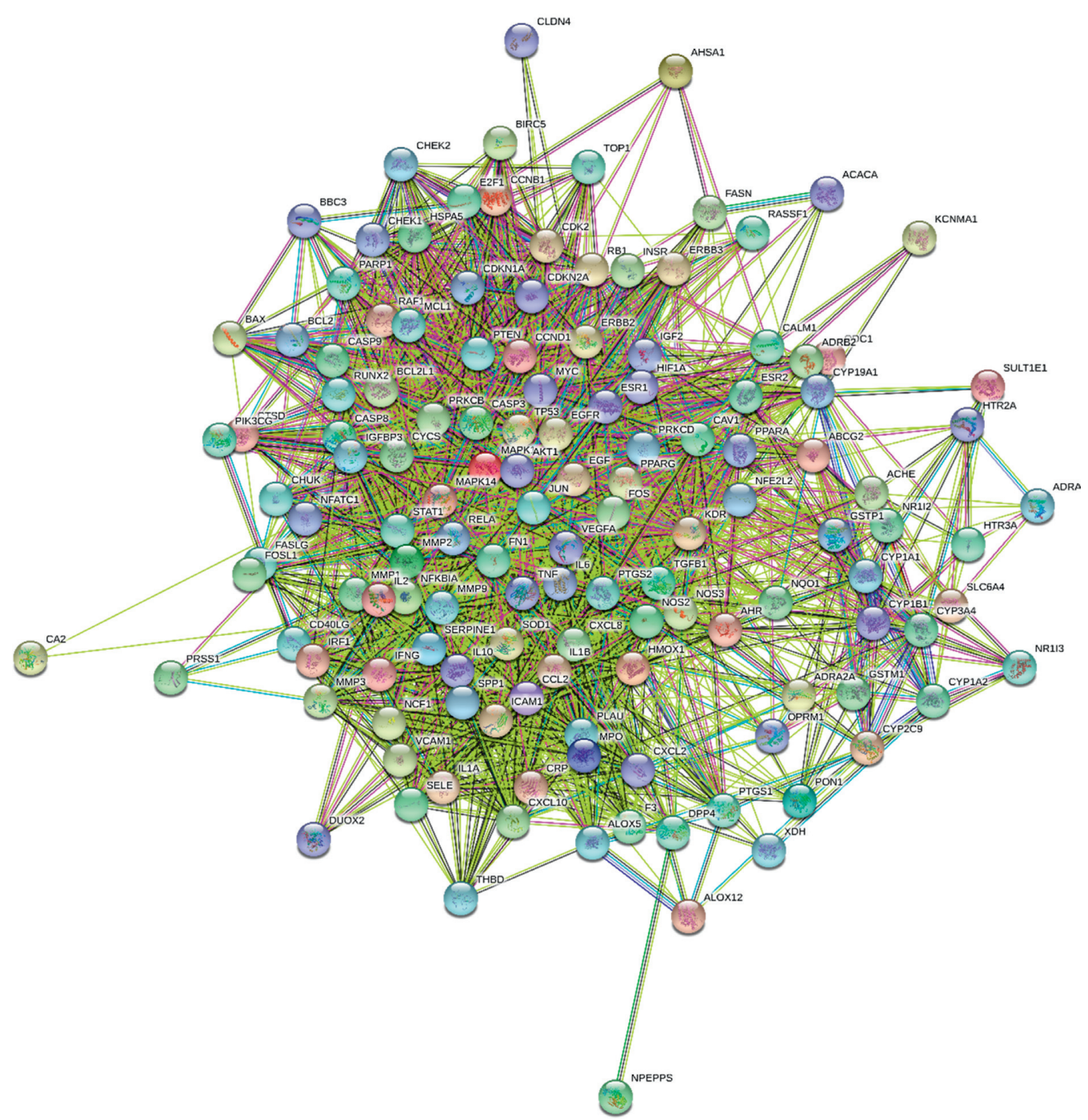

Figure 2: Common targets in the PPI network of SCDP for the treatment of ulcerative colitis. The PPI network includes 134 nodes, 2612 edges, and an average node degree of 39. PPI: protein-protein interaction; SCDP: Scutellariae radix-Coptidis rhizoma drug pair.

3.5. GO and KEGG Enrichment Analyses. Metascape was used for GO and KEGG analyses of the 134 hub genes to obtain enriched ontology clusters. As a result, we obtained 2304 GO terms, including 2073 biological processes (BPs), 79 cellular components (CCs), and 152 molecular functions (MFs). Figure 4 shows that BPs were mainly related to cytokine-mediated signaling pathway, response to inorganic substances, response to toxic substances, response to lipopolysaccharides (LPS), reactive oxygen species (ROS) metabolic processes, positive regulation of cell death, apoptotic signaling pathway, and response to wounding. The CCs were mainly related to membrane raft and transcription factor complex. Moreover, the MFs were mainly related to protein kinase binding, transcription factor binding, cytokine receptor binding, and protein homodimerization activity (Figure 4).

KEGG pathway enrichment analysis screening resulted in 164 signaling pathways. Visual analysis of the top 20 pathways showed that the main signaling pathways of SCDP in the treatment of UC were concentrated in pathways in cancer, the advanced glycation end products and the receptor for AGEs (AGE-RAGE) signaling pathway in diabetic complications, bladder cancer, IL-17 signaling pathway, apoptosis, p53 signaling pathway, and phosphoinositide 3kinase (PI3K)-Akt signaling pathway (Figure 5).

3.6. Drug Ingredient-Target-Pathway Interaction Network Construction. The Cytoscape software was used to construct an active ingredient-core target-key pathway network, which contained 41 nodes and 108 edges (Figure 6). According to the degree values (betweenness centralities and closeness centralities in the drug ingredient-target-pathway network), the main active ingredients of SCDP for UC treatment were determined to be quercetin, wogonin, baicalein, acacetin, oroxylin A, and beta-sitosterol, with the main targets being PTGS2, CASP3, TP53, IL-6, TNF, and AKT1 (Tables 5 and 6).

3.7. Molecular Docking. The molecular docking results indicate binding energies of less than $-2 \mathrm{kcal} / \mathrm{mol}$ after docking, suggesting the formation of stable bonds between 

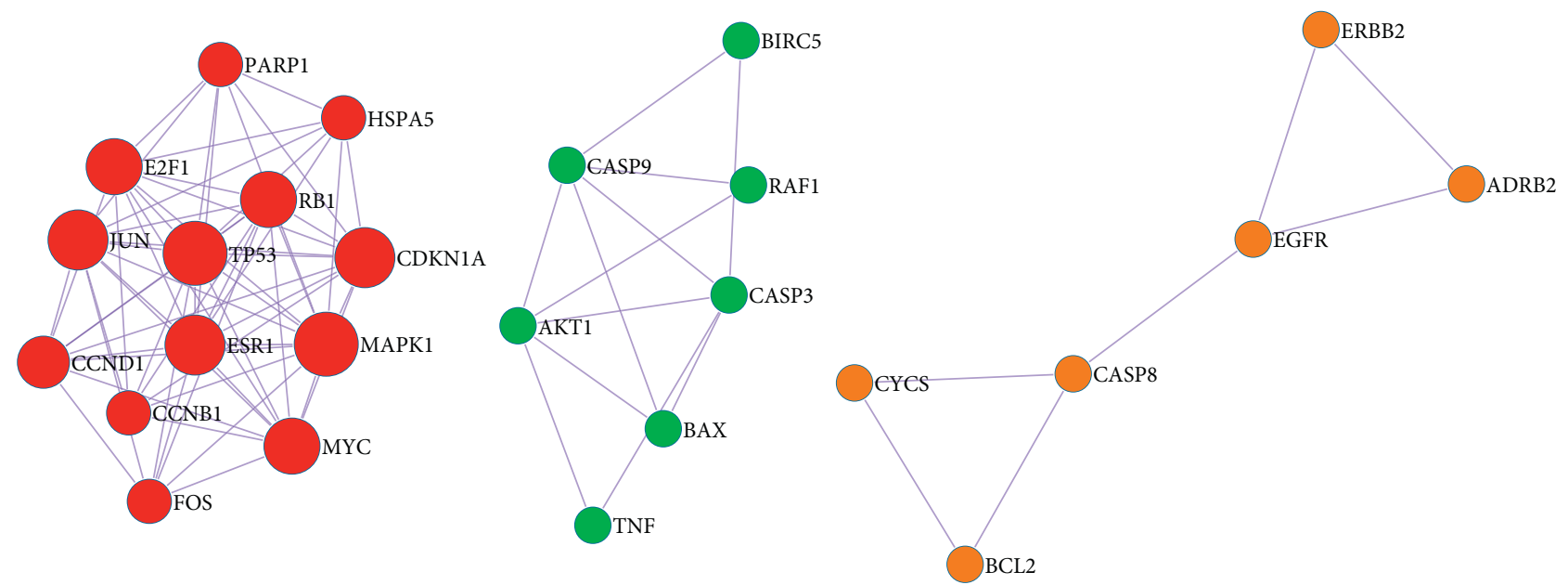

(a)

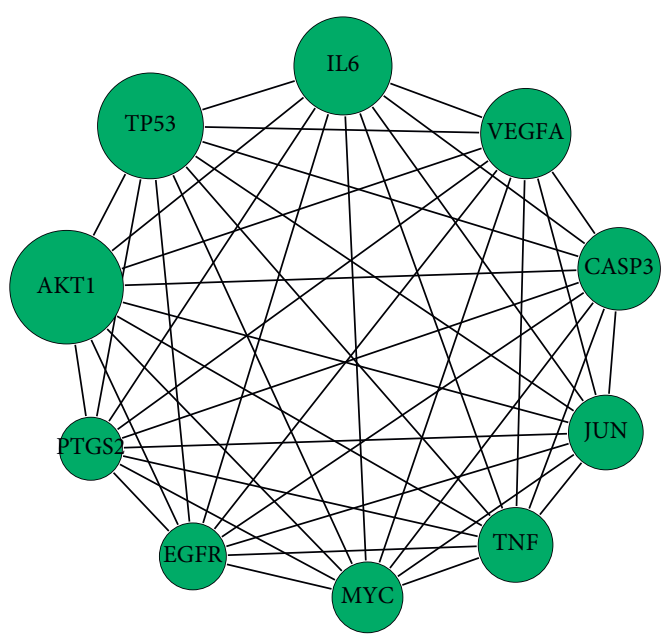

(b)

FIgURE 3: The core target PPI network module clusters (a) and core protein network diagram of SCDP for the treatment of ulcerative colitis (b). PPI: protein-protein interaction; SCDP: Scutellariae radix-Coptidis rhizoma drug pair.

TABle 4: The main targets of Scutellariae radix-Coptidis rhizoma drug pair.

\begin{tabular}{lccc}
\hline Rank & Gene symbol & Gene name & Degree \\
\hline 1 & AKT1 & RAC-alpha serine/threonine-protein kinase & 108 \\
2 & TP53 & Cellular tumor antigen p53 & 98 \\
3 & IL-6 & Interleukin-6 & 94 \\
4 & VEGFA & Vascular endothelial growth factor A & 93 \\
5 & CASP3 & Caspase-3 & 88 \\
6 & JUN & Transcription factor AP-1 & 86 \\
6 & TNF & Tumor necrosis factor & 86 \\
8 & MYC & Myc proto-oncogene protein & 85 \\
9 & EGFR & Epidermal growth factor receptor & 84 \\
10 & PTGS2 & Prostaglandin G/H synthase 2 \\
\hline
\end{tabular}




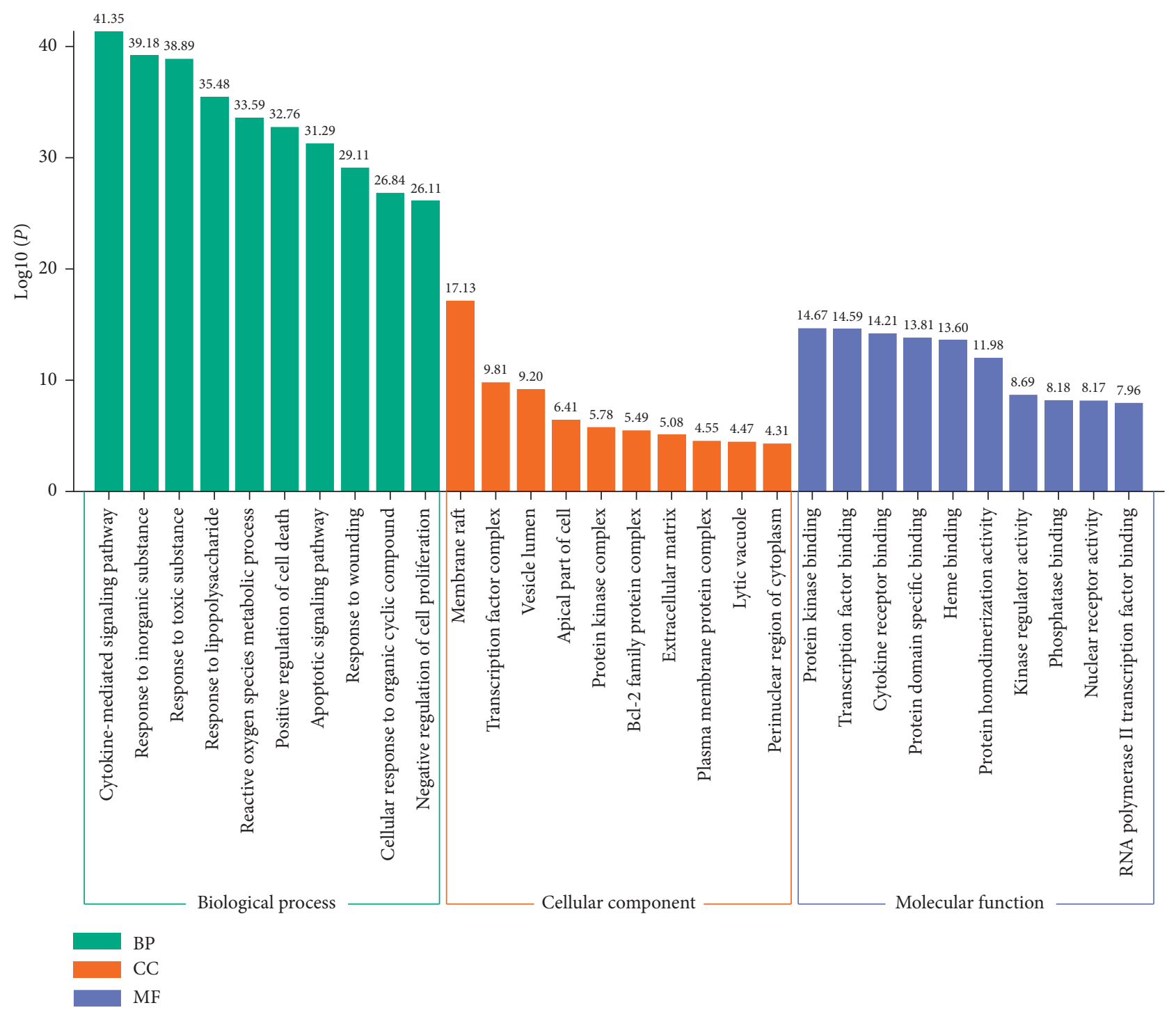

FIGURE 4: GO terms of candidate targets of SCDP against ulcerative colitis. The top 10 GO functional categories were selected. GO: gene ontology; SCDP: Scutellariae radix-Coptidis rhizoma drug pair; BP: biological process; CC: cellular component; MF: molecular function.

the active ingredients and predicted target proteins (Table 7). The PyMOL software was used to visualize the docking results of key active ingredients and core targets, as shown in Figure 7.

\section{Discussion}

UC is a type of irritable bowel syndrome (IBS), and the main pathogenesis of UC is spleen deficiency as the original cause and damp-heat with stasis toxin as the superficial factors in TCM theory [28-30]. Based on the theory of TCM, UC consists of several types of syndromes, the most common of which $(34.8 \%)$ being damp-heat accumulation syndrome $[4,31]$. Therefore, the main treatment approach is to enhance the spleen Qi and eliminate damp-heat toxin. Dampheat and stasis toxin are important factors in the recurrence of this disease [32].

SCDP is a commonly used drug pair for eliminating damp-heat toxin. These two herbs are also constituents of many classical formulas, such as decoctions Gegen Qinlian, Huangqin, Shaoyao, Baitouweng, Huanglian Jiedu, Banxia Xiexin, and WuMei pill, for the treatment of diarrhea as a symptom of UC, by TCMs [27].

The damp-heat insidious pathogen has been proposed as the mechanism responsible for UC recurrence, necessitating that damp-heat toxin is cleared for UC treatment. In turn, Cao et al. advocated the use of Scutellariae radix, Coptidis rhizoma, Cortex Phellodendri, Sophora flavescens, pulsatilla, ash bark, among others, in combination with dampnessdissipating drugs, such as Pogostemon cablin and eupatorium, while Y. Zhang et al. suggested the use of Coptidis rhizoma, Scutellariae radix, Cortex Phellodendri, Sophora flavescens, and pulsatilla as the main ingredients in the formulation $[27,33,34]$. To further clarify the internal mechanism of SCDP for UC treatment, we applied network pharmacology to analyze its active ingredients, targets, and pathways, thus providing ideas for future research and drug development. 


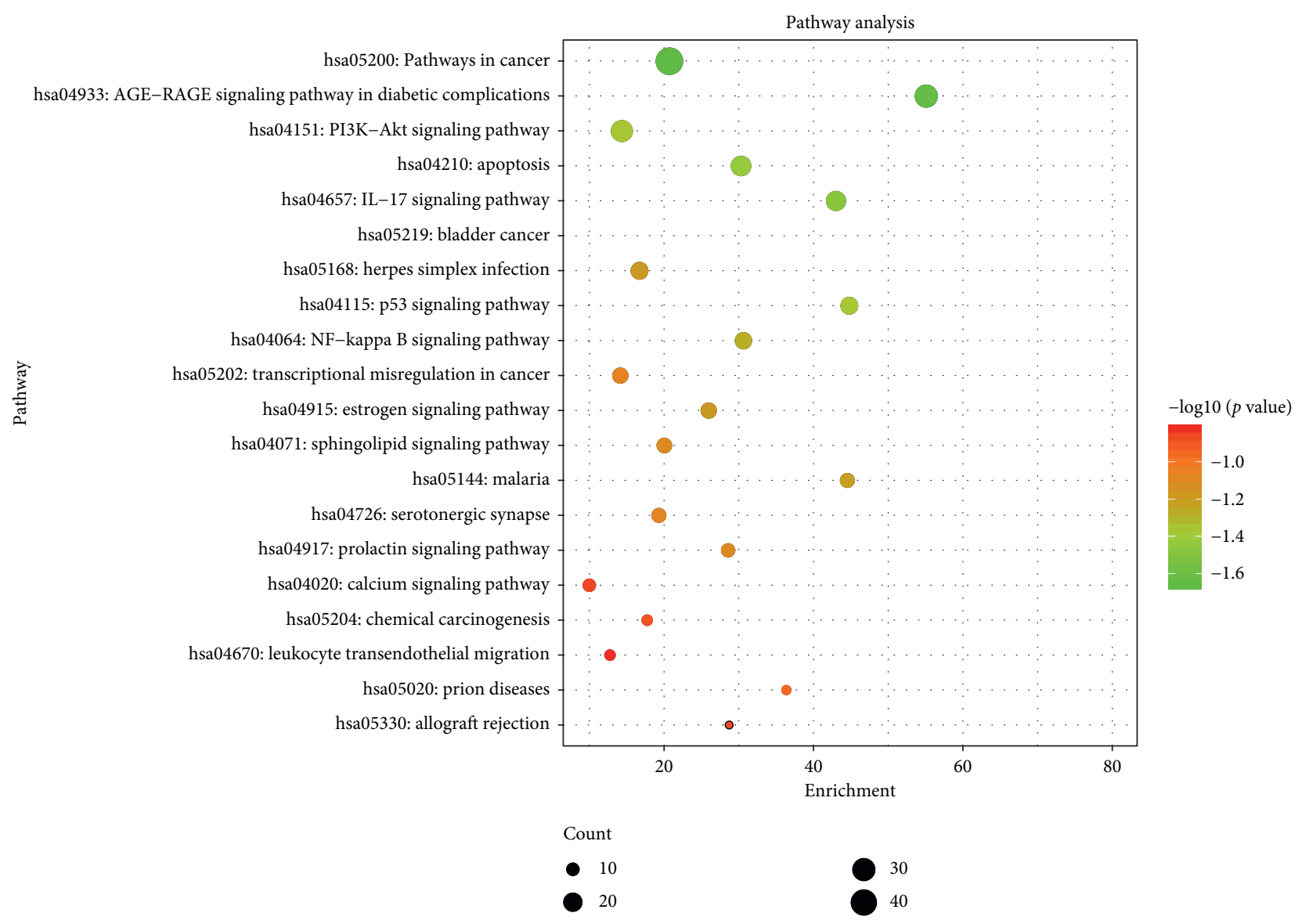

FIGURE 5: KEGG pathway enrichment of top 20 pathways of SCDP in the treatment of ulcerative colitis. The size of the spot represents the number of genes, and the color represents enrichment of $-\log 10$ ( $p$ value). KEGG: Kyoto Encyclopedia of Genes and Genomes; SCDP: Scutellariae radix-Coptidis rhizoma drug pair.

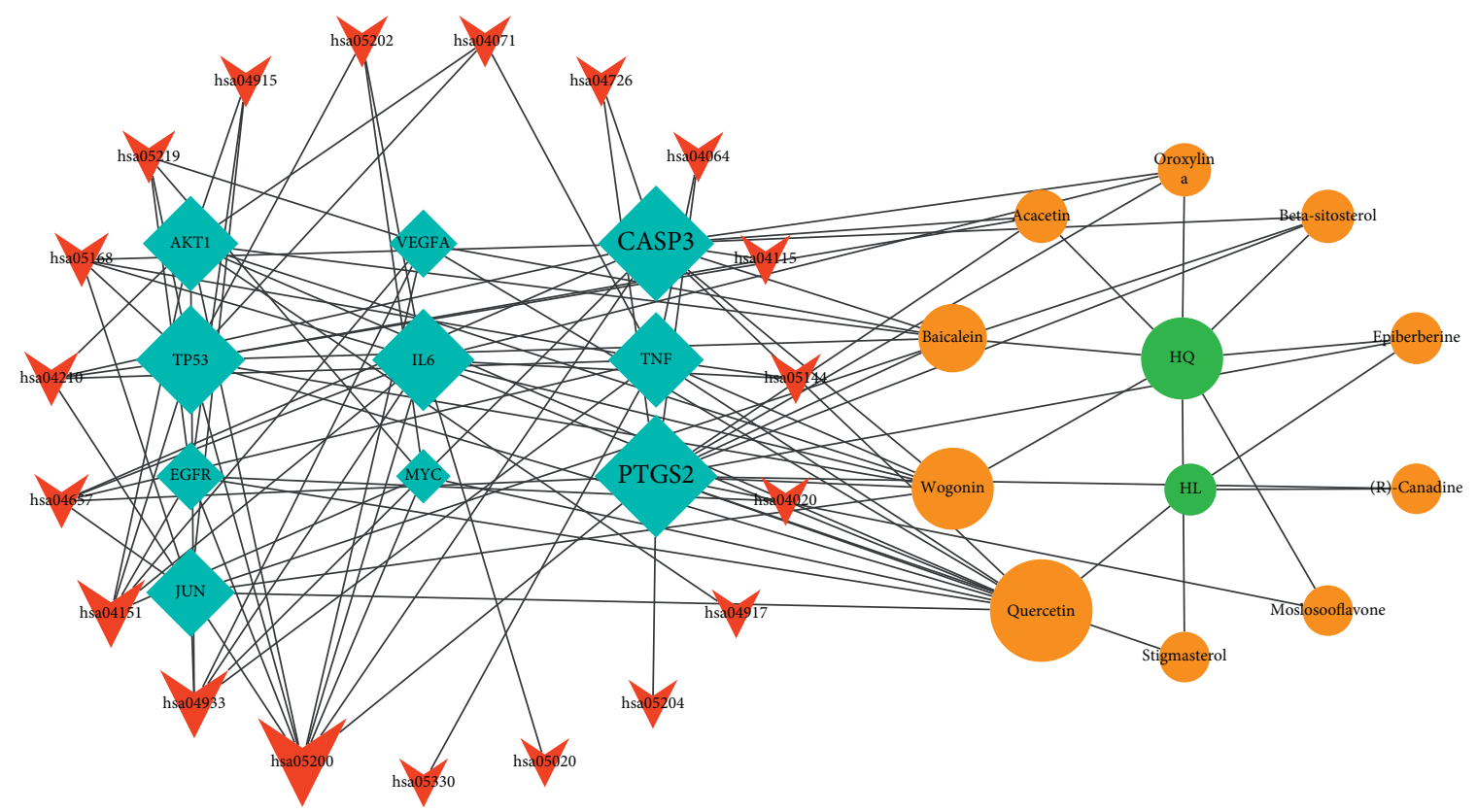

FIGURE 6: Key ingredient-protein target-pathway network for SCDP in the treatment of ulcerative colitis. Green circles represent the Scutellariae radix as "HQ" and Coptidis rhizoma as "HL." Orange circle represents key active ingredients, while blue diamond represents key protein targets. The red V-shape represents key pathways from KEGG enrichment analysis. The area and color transparency of the node represent its degree; the darker the area and color, the more important the node. SCDP: Scutellariae radix-Coptidis rhizoma drug pair. 
TABLE 5: Key ingredients obtained from ingredient-target-pathway network.

\begin{tabular}{lcccc}
\hline Molecule ID & Name & Degree $\geq 4$ & Betweenness centrality & Closeness centrality \\
\hline MOL000098 & Quercetin & 11 & 0.1762 & 0.5714 \\
MOL000173 & Wogonin & 8 & 0.0789 & 0.5128 \\
MOL002714 & Baicalein & 6 & 0.0405 & 0.4545 \\
MOL001689 & Acacetin & 4 & 0.0145 & 0.4167 \\
MOL002928 & Oroxylin A & 4 & 0.0171 & 0.4167 \\
MOL000358 & Beta-sitosterol & 4 & 0.0114 & 0.3922 \\
\hline
\end{tabular}

TABLE 6: Core targets obtained from ingredient-target-pathway network.

\begin{tabular}{lccc}
\hline Name & $\begin{array}{c}\text { Degree } \\
\geq 10\end{array}$ & $\begin{array}{c}\text { Betweenness } \\
\text { centrality }\end{array}$ & $\begin{array}{c}\text { Closeness } \\
\text { centrality }\end{array}$ \\
\hline PTGS2 & 15 & 0.2576 & 0.5063 \\
CASP3 & 13 & 0.1119 & 0.4819 \\
TP53 & 12 & 0.1144 & 0.4706 \\
IL-6 & 11 & 0.1265 & 0.4598 \\
TNF & 10 & 0.1098 & 0.4494 \\
AKT1 & 10 & 0.1007 & 0.4494 \\
\hline
\end{tabular}

TABle 7: Binding energy between key ingredients and target proteins.

\begin{tabular}{lcccccc}
\hline \multirow{2}{*}{ Key ingredients } & \multicolumn{7}{c}{ Binding energy (kcal/mol) } \\
& PTGS2 & CASP3 & TP53 & IL-6 & TNF & AKT1 \\
\hline Quercetin & -2.25 & -1.94 & -1.74 & -1.60 & -1.73 & -2.90 \\
Wogonin & -2.88 & -2.57 & -3.22 & -1.72 & -3.10 & -2.44 \\
Baicalein & -4.78 & -2.33 & -2.4 & -2.16 & -3.38 & -2.53 \\
Acacetin & -4.66 & -2.59 & -2.74 & -2.46 & -2.78 & -2.46 \\
Oroxylin A & -2.95 & -2.54 & -3.09 & -2.11 & -2.94 & -2.51 \\
Beta-sitosterol & -2.34 & -2.23 & -1.95 & -1.49 & -2.80 & -3.16 \\
\hline
\end{tabular}

The results show that the key active ingredients are quercetin, wogonin, baicalein, acacetin, oroxylin $\mathrm{A}$, and beta-sitosterol. Previous research discovered that quercetin inhibits the expression of LPS-induced inflammatory genes, mainly by reducing the levels of TNF- $\alpha$ and lipocalin- 2 mRNA and enhancing the expression of the Slip protein to protect the UC mucosa [35]. It has been found that quercetin exerts a therapeutic effect in IBD by regulating the structure of the intestinal flora, inhibiting the expression of proinflammatory factors, such as IL-17, TNF- $\alpha$, IL-6, and increasing the expression of IL-10 [36].

Wogonin has strong anti-inflammatory, antitumor, antiviral and antiallergic properties. It can inhibit the activity of Treg cells, regulate the differentiation of Th17 cells, and inhibit the transcription of the extracellular signal-regulated kinase (ERK), signal transducer and activator of transcription 3 (STAT3), and hypoxia-inducible factor- $1 \alpha$ to regulate inflammatory responses [37]. One study found that $25-\mathrm{mg} / \mathrm{mL}$ wogonin can reduce the LPS-induced inflammatory response of the TLR4-MyD88-mediated NF- $\kappa \mathrm{B}$ pathway in Caco-2 cells, suggesting its protective effect on the intestinal mucosal barrier [38].

Baicalein is the main ingredient of Coptidis rhizoma. Studies have suggested its anti-inflammatory effect and discovered that it could improve the inflammatory state of UC and the control drugs [39-41]. The effect of baicalein is similar to that of the reference drug sulfasalazine and comparable to wogonin $[42,43]$. In addition, baicalein reduces the levels of the inflammatory mediators IL-33 and $\mathrm{NF}-\kappa \mathrm{B}$ p 65 and increases the level of $\mathrm{I} \kappa \mathrm{B} \alpha$ to resists the pathological changes of dextran sulfate sodium (DSS)-induced UC [44].

Acacetin (5,7-dihydroxy- $4^{\prime}$-methoxyflavone) is an O-methylated flavone naturally present in plants like chrysanthemum and safflower, as well as in Calamintha and Linaria species. Acacetin possesses antiperoxidative, antiinflammatory, antiplasmodial, and anticancer activities. In addition, acacetin strongly inhibits the expression of proinflammatory cytokines, inducible nitric oxide synthase (iNOS), and COX-2 in LPS-induced RAW 264.7 cells [45]. Research has shown that acacetin treatment could inhibit sepsis-induced acute lung injury and reduced iNOS and COX-2 expression.

Oroxylin A and baicalein are produced as major metabolites of baicalin by the action of bacteria in the large intestine cavity. In addition, both baicalin and baicalein are converted into four new metabolites: wogonin, wogonoside, oroxylin A, and oroxin A [46]. Some studies have shown that oroxylin A is involved in the induction of autophagy and apoptosis, potentially via its regulation of p62-mediated caspase- 8 , which promotes the transcription of NF- $\kappa \mathrm{B}$ downstream target genes and inhibits the transcription of downstream target genes of Nrf2 [47-49].

Beta-sitosterol was found to markedly reduce weight loss, colonic length shortening, and microscopic changes in DSS-induced colitis and to reduce TNF- $\alpha$, IL-6, and IL- $1 \beta$ levels in the intestinal tissues of experimental colitis mice in a concentration-dependent manner [50].

The core targets of SCDP in UC treatment are PTGS2 (COX2), CASP3, TP53, IL-6, TNF, and AKT1, which are associated with the TNF signaling pathway and NF- $\kappa$ B and oxidative stress pathways. An important target of anti-inflammatory drugs, especially nonsteroidal anti-inflammatory drugs, is prostaglandin endoperoxide synthase or COX. There are two subtypes of COX. COX1 is constitutively expressed and is responsible for regulating normal physiological processes. COX2 is induced under inflammatory conditions and mainly regulates the inflammatory process. It has been suggested that the expression of COX2 is induced in the colon of IBD patients but also in the inflamed tissues of IL-10-deficient IBD mouse models, and reduced COX2 is considered a major target for IBD treatment [46].

A previous study in UC rats revealed that baicalin could up-regulate B-cell lymphoma 2 (Bcl-2) protein expression, lower Bax (also known as Bcl-2-like protein 4) protein 


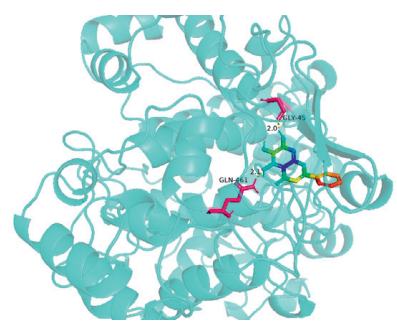

(a)

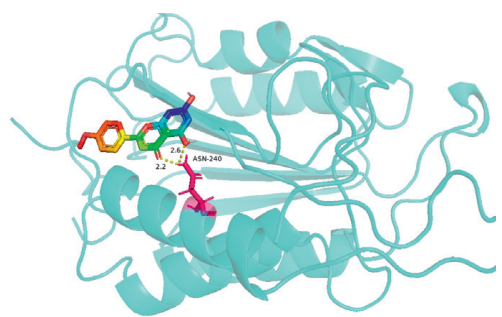

(d)

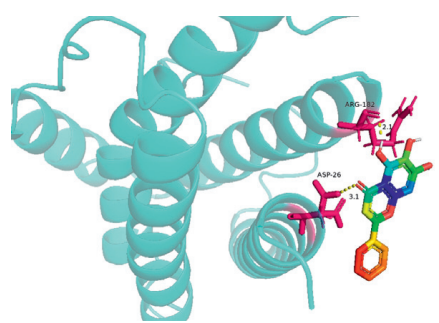

(g)

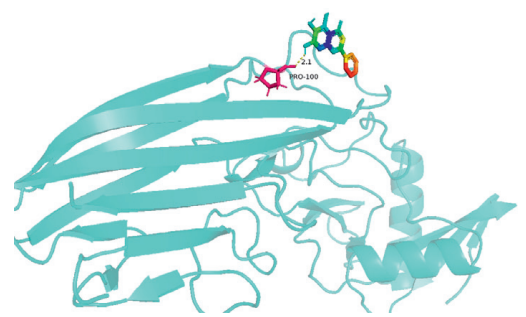

(j)

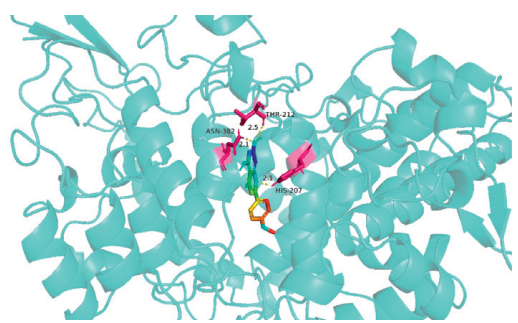

(b)

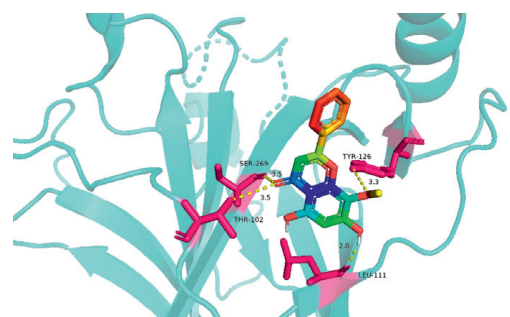

(e)

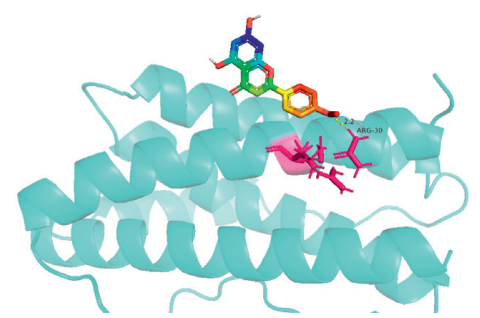

(h)

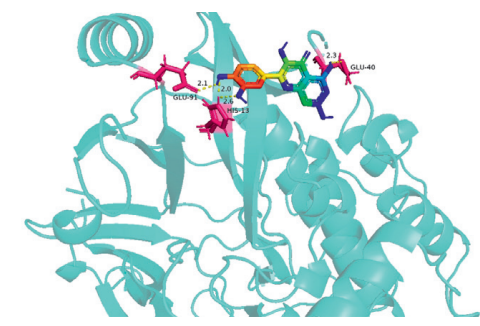

(k)

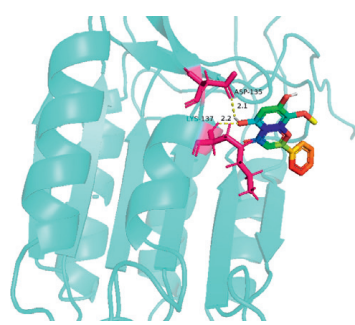

(c)

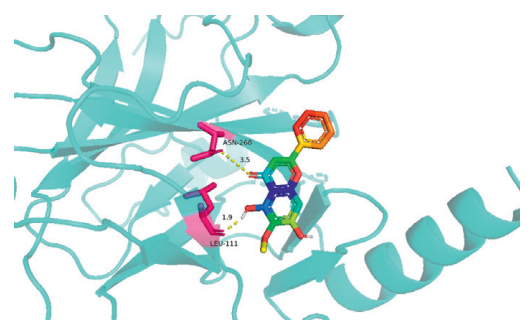

(f)

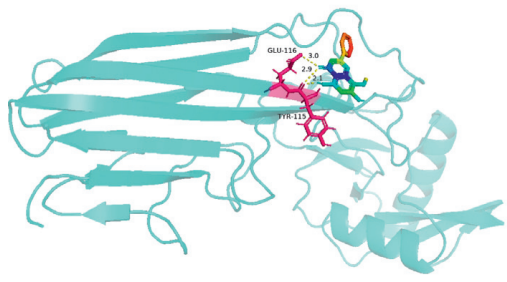

(i)

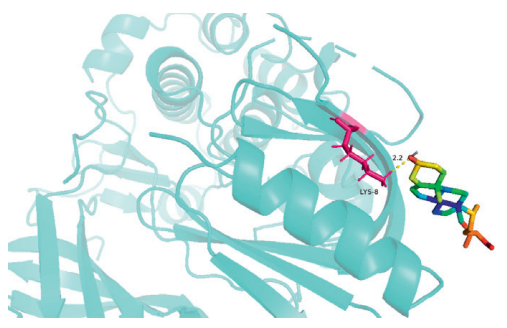

(l)

FIGURE 7: Docking results of the key ingredient and six hubs target proteins. (a) Baicalein and PTGS2; (b) acacetin and PTGS2; (c) wogonin and CASP3; (d) acacetin and CASP3; (e) wogonin and TP53; (f) oroxylin A and TP53; (g) baicalein and IL-6; (h) acacetin and IL-6; (i) wogonin and TNF; (j) baicalein and TNF; (k) quercetin and AKT1; (l) beta-sitosterol and AKT1.

expression, induce marked activation of the caspase-cascade system, and exhibit concentration-dependent down-regulation of Fas and Fas Ligand (FasL) protein expressions to exert therapeutic effects in UC [10]. AKT1 is a key mediator of the PI3K-Akt signaling pathway and is involved in cellular function regulation in various tumors, including gastric cancer, glioma, lung cancer, and esophageal squamous cell carcinoma [51, 52].

Berberine has been found to play a regulatory role in macrophage M1 polarization in DSS-induced colitis through the AKT1-suppressor of cytokine signaling-1 (SOCS1)-NF$\kappa \mathrm{B}$ signaling pathway [53]. IL-6 gene expression is strongly associated with IBD progression [54]. JUN is a proto-oncogene that plays a key role in inflammation, and can be activated by many inflammatory factors directly or indirectly. The activation of JUN further modulates the expression and regulation of relevant inflammatory factors, which, in turn, participate in the regulation of the inflammatory response [55]. Being a proinflammatory regulator, TNF promotes the release of proinflammatory factors and plays an important role in the development and progression of UC. In addition, together with interferons, it alters the barrier function of intestinal epithelial cells, enhances the permeability of the intestinal mucosa and vascular wall, and disrupts the integrity of the intestinal mucosa, leading to ulcer formation [56, 57].

GO analysis and KEGG enrichment results showed that the mechanism of SCDP in the treatment of UC was mainly related to the following pathways: pathways in cancer, PI3KAkt signaling pathway, the AGE-RAGE signaling pathway in diabetic complications, apoptosis, IL-17 signaling pathway, and herpes simplex infection.

According to Rogler, up to $30 \%$ of the patients with chronic active UC or Crohn's disease (CD) are likely to get 
colorectal cancer after 35 years since the onset of the disease [58]. Chronic inflammation, the increased turnover of epithelial cells, and ROS production are thought to contribute to the development of dysplastic lesions, which may then transform into colorectal cancer [59]. Meanwhile, chronic UC status is also a major risk factor in the development of colitis-associated cancer [59].

IL-17, mainly secreted by Th17 cells, is involved in inflammatory response, and its levels have been confirmed to correlate with the extent of UC disease. Acting as a proinflammatory factor, IL-23 promotes IL-17 production and forms the IL-23/17 axis to amplify the inflammatory response $[60,61]$. IL-17A induces the aggregation of neutrophils on target cells, resulting in the secretion of inflammatory cytokines (IL-6, TNF- $\alpha$, and IL-1B), chemokines (CXC), granulocyte colony-stimulating factor, and granulocyte-macrophage colony-stimulating factor. Meanwhile, IL-17A is also involved in the regulation of mucosal barrier function and amplification of the complete inflammatory response through the production of antimicrobial peptides, such as $\beta$-defensins, S100 $\beta$-defensins, and S100 proteins [62]. The strongest evidence comes from the genome-wide association studies that have identified IBD susceptibility single nucleotide polymorphisms in many genes involved in the IL-23/IL-17 axis $[63,64]$. Other susceptibility genes in Crohn's disease and UC also appear to be involved in the IL23/IL-17 axis. For example, STAT3, janus kinase 2 (JAK2) and tyrosine kinase 2 (TYK2) participate in IL-23 signaling, and IL-12B encodes the common subunit of IL-12 and IL-23 [61].

The mutation of the p53 gene is an important genetic alteration involved in the early stages of UC-associated colorectal cancer. The overexpression of $\mathrm{p} 53$ protein in the colorectal crypt of UC patients, usually in the absence of atypical hyperplasia observation of atypical hyperplasia, is used by pathologists to define the state between regenerative changes and intraepithelial neoplasia. It has been used as a biomarker in predicting the risk of progression into a malignant tumor. High-frequency mutations of p53 were reported in severe chronic UC patients with undiagnosed cancer [65]. The expression of p53 in UC patients showed a negative correlation with IL-6, which is mainly involved in the development of cancer-induced by chronic inflammation. IL- 6 is the main activator of STAT3, and its stimulation products increase the transcription of target genes, as well as cell proliferation/survival. Downregulation of p53 expression is probably the most important step in the transformation of inflamed tissues and cells. Particularly, inflammatory cells that stimulate proliferation and inhibit apoptosis of cells, cytokines, chemokines, and growth factors produce ROS and reactive nitrogen species that cause oxidative DNA damage. The damage may not be adequately repaired because the $\mathrm{p} 53$ function is downregulated by IL- 6 [66].

In addition, TCMs may exert relevant apoptotic inhibitory effects through the upregulation of p53 and Bcl-2 expression, which is accompanied by the downregulation of TNF- $\alpha$ and IL- 1 and the upregulation of IL- 6 and IL-10. The PI3K-AKT signaling pathway is involved in the regulation and release of proinflammatory cytokines, including TNF- $\alpha$ and other cytokines closely related to the inflammatory response of UC [67], and plays an important role in the development and progression of UC. TCMs inhibit transforming growth factor- $\beta$ (TGF- $\beta$ ) expression and lead to a decrease in PI3K/AKT activation, thereby alleviating the symptoms of UC by regulating TGF- $\beta$ expression [68]. AGE and RAGE are involved in and mediate various signaling pathways of oxidative stress, induce ROS production, and activate NF- $\kappa \mathrm{B}$, leading to inflammatory responses, cell apoptosis, and microvascular diseases [69]. RAGE polymorphisms and increased RAGE levels have been found in IBD patients, and the involvement of AGE/RAGE in inflammation correlates with its activation of NF- $\kappa \mathrm{B}$ and its response to oxidative stress [70].

The results of molecular docking showed that quercetin, wogonin, baicalein, acacetin, oroxylin A, and beta-sitosterol, the main active ingredients of SCDP, had a good affinity to the core target genes PTGS2, CASP3, TP53, IL-6, TNF, and $A K T 1$, suggesting that the key active ingredients of SCDP play a therapeutic role in UC by mainly intervening with the key signaling targets, such as the inflammatory response and oxidative stress. In summary, the potential biological mechanism of SCDP in the treatment of UC involves multiple ingredients, targets, and pathways, and the therapeutic effects occur through several pathways as pathways in cancer, PI3K-Akt signaling pathway, apoptosis, and IL-17 signaling pathway.

The presented study provides a scientific basis for the subsequent development and utilization of SCDP. Even so, network pharmacology has only analyzed the main active ingredients and targets of drugs, and further validation of their predicted target pathways is needed experimentally. The conclusions of this research also contribute to the research and development of drugs and further elaboration of mechanisms.

\section{Data Availability}

All data are fully available without restriction, and all relevant data are included within the paper.

\section{Disclosure}

The funders had no role in study design, in the collection, analysis, and interpretation of data, in the writing of the report, and in the decision to submit the article for publication.

\section{Conflicts of Interest}

The authors declare that there are no conflicts of interest regarding the publication of this article.

\section{Authors' Contributions}

Kai Niu and Qifang Li contributed equally to this work. B. Y. conceived and designed the study. Q. L., K. N., and K. W. developed the methodology. Q. L., L. C., C. W., Y. L., and B. L. performed the data acquisition. C. Z., R. W., H. Z., C. 
W., L. Z., B. S., and B. L. performed data analysis and interpretation. Y. Q. and B. Y. drafted, reviewed, and revised the manuscript. All authors read and approved the final manuscript.

\section{Acknowledgments}

This work was supported by the National Natural Science Foundation of China (No. 81603509), the Natural Science Foundation of Shandong Province (No. ZR2014HQ051), and the College Students' Innovation and Entrepreneurship Training Program of Jining Medical University (No. cx2020067).

\section{References}

[1] I. Khan, N. Ullah, L. Zha et al., "Alteration of gut microbiota in inflammatory bowel disease (IBD): cause or consequence? IBD treatment targeting the Gut microbiome," Pathogens, vol. 8, no. 3, 2019.

[2] S. C. Ng, W. K. Leung, H. Y. Shi et al., "Epidemiology of inflammatory bowel disease from 1981 to 2014: results from a territory-wide population-based registry in Hong Kong," Inflammatory Bowel Diseases, vol. 22, no. 8, pp. 1954-1960, 2016.

[3] S. C. Ng, C. N. Bernstein, M. H. Vatn et al., "Geographical variability and environmental risk factors in inflammatory bowel disease," Gut, vol. 62, no. 4, pp. 630-649, 2013.

[4] M. Chen, Y. Ding, and Z. Tong, "Efficacy and safety of Sophora flavescens (kushen) based traditional Chinese medicine in the treatment of ulcerative colitis: clinical evidence and potential mechanisms," Frontiers in Pharmacology, vol. 11, 2020.

[5] S. C. Ng, H. Y. Shi, N. Hamidi et al., "Worldwide incidence and prevalence of inflammatory bowel disease in the 21st century: a systematic review of population-based studies," The Lancet, vol. 390, no. 10114, pp. 2769-2778, 2017.

[6] D. R. Plichta, D. B. Graham, S. Subramanian, and R. J. Xavier, "Therapeutic opportunities in inflammatory bowel disease: mechanistic dissection of host-microbiome relationships," Cell, vol. 178, no. 5, pp. 1041-1056, 2019.

[7] M. F. Neurath, "Cytokines in inflammatory bowel disease," Nature Reviews Immunology, vol. 14, no. 5, pp. 329-342, 2014.

[8] Y. N. Wang, J. Li, W. Y. Zheng et al., "Clinical characteristics of ulcerative colitis-related colorectal cancer in Chinese patients," Journal of Digestive Diseases, vol. 18, no. 12, pp. 684-690, 2017.

[9] M. Duijvestein, R. Battat, N. Vande Casteele et al., "Novel therapies and treatment strategies for patients with inflammatory bowel disease," Curr Treat Options Gastroenterol, vol. 16, no. 1, pp. 129-146, 2018.

[10] J. Yao, X. Cao, R. Zhang et al., "Protective effect of baicalin against experimental colitis via suppression of oxidant stress and apoptosis," Pharmacognosy Magazine, vol. 12, no. 47, pp. 225-234, 2016.

[11] H. W. Lee, J. H. Jun, K.-J. Kil et al., "Herbal medicine (Danggui Shaoyao San) for treating primary dysmenorrhea: a systematic review and meta-analysis of randomized controlled trials," Maturitas, vol. 85, pp. 19-26, 2016.

[12] Z. Lu, W. Xiong, S. Xiao et al., "Huanglian Jiedu decoction ameliorates DSS-induced colitis in mice via the JAK2/STAT3 signalling pathway," Chinese Medicine, vol. 15, p. 45, 2020.

[13] X. Ke, Y. Huang, L. Li et al., "Berberine attenuates arterial plaque formation in atherosclerotic rats with damp-heat syndrome via regulating autophagy," Drug Design, Development and Therapy, vol. 14, pp. 2449-2460, 2020.

[14] X. Xie, X. Ma, S. Zeng et al., "Mechanisms of berberine for the treatment of atherosclerosis based on network pharmacology," Evidence-based Complementary and Alternative Medicine: ECAM, vol. 2020, Article ID 3568756, 11 pages, 2020.

[15] R. Li, Y. Chen, M. Shi et al., "Gegen Qinlian decoction alleviates experimental colitis via suppressing TLR4/NF- $\kappa \mathrm{B}$ signaling and enhancing antioxidant effect," Phytomedicine, vol. 23, no. 10, pp. 1012-1020, 2016.

[16] Y. Wan, L. Xu, Z. Liu et al., "Utilising network pharmacology to explore the underlying mechanism of Wumei Pill in treating pancreatic neoplasms," BMC Complementary and Alternative Medicine, vol. 19, no. 1, p. 158, 2019.

[17] Q. Ji, Y. Yang, X. Song, X. Han, and W. Wang, "Banxia Xiexin Decoction in the treatment of chronic atrophic gastritis," Medicine, vol. 99, no. 42, Article ID e22110, 2020.

[18] N. Zhu, J. Hou, G. Ma, and J. Liu, "Network pharmacology identifies the mechanisms of action of Shaoyao gancao decoction in the treatment of osteoarthritis," Medical Science Monitor, vol. 25, pp. 6051-6073, 2019.

[19] Z. Hu, Y. Zhang, W. Zheng, H. Wang, and X. Sun, “Analysis of the medication rule and mechanism of patent-prescription of Chinese medicine for ulcerative colitis based on patent database," Chinese Journal of Hospital Pharmacy, vol. 40, no. 17, pp. 1811-1815, 2021.

[20] W. Shan, J. Xie, and M. Li, "Xie Jingri's experience in the treatment of ulcerative colitis with drug-pairs," Journal of Changchun University of Chinese Medicine, vol. 36, no. 3, pp. 451-453, 2019.

[21] H. Li, Y. Xie, Y. Zhu et al., "Literature study on medication regularity and prescription-formulating principle of Chinese medicine for treatment of ulcerative colitis," Journal of Guangzhou University of Traditional Chinese Medicine, vol. 37, no. 1, pp. 176-181, 2020, in Chinese.

[22] H. Li, Y. Xie, Q. Li et al., "Analysis of prescription rules in treating ulcerative colitis with liver-spleen disorder syndrome based on traditional Chinese medicine inheritance support system," China Medicine, vol. 15, no. 10, pp. 1580-1584, 2020, in Chinese.

[23] S. Yan, L. Yingchao, W. Zhangliu et al., "Effect of berberine from Coptis chinensis on apoptosis of intestinal epithelial cells in a mouse model of ulcerative colitis: role of endoplasmic reticulum stress," Evidence-based Complementary and Alternative Medicine: ECAM, vol. 2020, Article ID 3784671, 7 pages, 2020.

[24] J. Deng, Z. Wu, Z. Zhao et al., "Berberine-loaded nanostructured lipid carriers enhance the treatment of ulcerative colitis," International Journal of Nanomedicine, vol. 15, pp. 3937-3951, 2020.

[25] F. Cheng, I. A. Kovács, and A.-L. Barabási, "Network-based prediction of drug combinations," Nature Communications, vol. 10, no. 1, p. 1197, 2019.

[26] Q. Tao, J. Du, X. Li et al., "Network pharmacology and molecular docking analysis on molecular targets and mechanisms of Huashi Baidu formula in the treatment of COVID19," Drug Development and Industrial Pharmacy, vol. 46, no. 8, pp. 1345-1353, 2020.

[27] Z. Shen, Q. Zhou, Y. Ni, W. He, H. Shen, and L. Zhu, "Traditional Chinese medicine for mild-to-moderate ulcerative colitis," Medicine, vol. 98, no. 33, Article ID e16881, 2019.

[28] A. Imdad, M. R. Nicholson, E. E. Tanner-Smith et al., "Fecal transplantation for treatment of inflammatory bowel disease," 
The Cochrane Database of Systematic Reviews, vol. 11, Article ID CD012774, 2018.

[29] Z.-H. Shen, C.-X. Zhu, Y.-S. Quan et al., "Relationship between intestinal microbiota and ulcerative colitis: mechanisms and clinical application of probiotics and fecal microbiota transplantation," World Journal of Gastroenterology, vol. 24, no. 1, pp. 5-14, 2018.

[30] M. Wei, H. Li, Q. Li et al., "Based on network pharmacology to explore the molecular targets and mechanisms of gegen Qinlian decoction for the treatment of ulcerative colitis," BioMed Research International, vol. 2020, p. 5217405, 2020.

[31] B. Ye, H. Shen, Y. Lu, and Y.-Q. Wang, "Clinical observations on 100 cases of ulcerative colitis treated with the method of clearing away heat, expelling dampness, promoting blood circulation and healing ulcer," Journal of Traditional Chinese Medicine, vol. 30, no. 2, pp. 98-102, 2010.

[32] B. Liu, X. Piao, W. Niu et al., "Kuijieyuan decoction improved intestinal barrier injury of ulcerative colitis by affecting TLR4dependent PI3K/AKT/NF- $\kappa \mathrm{B}$ oxidative and inflammatory signaling and gut microbiota," Frontiers in Pharmacology, vol. 11, p. 1036, 2020.

[33] Y. Zhang, X. Li, X. Xu, and N. Yang, "Mechanisms of paeonia lactiflora in treatment of ulcerative colitis: a network pharmacological study," Medical Science Monitor, vol. 25, pp. 7574-7580, 2019.

[34] S.-Y. Cao, S.-J. Ye, W.-W. Wang, B. Wang, T. Zhang, and Y.-Q. Pu, "Progress in active compounds effective on ulcerative colitis from Chinese medicines," Chinese Journal of Natural Medicines, vol. 17, no. 2, pp. 81-102, 2019.

[35] M. Dicarlo, G. Teti, G. Verna et al., "Quercetin exposure suppresses the inflammatory pathway in intestinal organoids from Winnie mice," International Journal of Molecular Sciences, vol. 20, no. 22, 2019.

[36] R. Lin, M. Piao, and Y. Song, "Dietary quercetin increases colonic microbial diversity and attenuates colitis severity in Citrobacter rodentium-infected mice," Frontiers in Microbiology, vol. 10, p. 1092, 2019.

[37] L. Fan, D. Qiu, G. Huang et al., "Wogonin suppresses IL-10 production in B cells via STAT3 and ERK signaling pathway," Journal of Immunology Research, vol. 2020, Article ID 3032425, 12 pages, 2020.

[38] W. Wang, T. Xia, and X. Yu, "Wogonin suppresses inflammatory response and maintains intestinal barrier function via TLR4-MyD88-TAK1-mediated NF- $\kappa \mathrm{B}$ pathway in vitro," Inflammation Research, vol. 64, no. 6, pp. 423-431, 2015.

[39] W. Lee, S.-K. Ku, and J.-S. Bae, "Anti-inflammatory effects of baicalin, baicalein, and wogonin in vitro and in vivo," Inflammation, vol. 38, no. 1, pp. 110-125, 2015.

[40] C. Liu, Y. Li, Y. Chen et al., "Baicalein restores the balance of Th17/treg cells via aryl hydrocarbon receptor to attenuate colitis," Mediators of Inflammation, vol. 2020, Article ID 5918587, 19 pages, 2020.

[41] B. Dinda, S. Dinda, S. DasSharma, R. Banik, A. Chakraborty, and M. Dinda, "Therapeutic potentials of baicalin and its aglycone, baicalein against inflammatory disorders," European Journal of Medicinal Chemistry, vol. 131, pp. 68-80, 2017.

[42] A. Salaritabar, B. Darvishi, F. Hadjiakhoondi et al., "Therapeutic potential of flavonoids in inflammatory bowel disease: a comprehensive review," World Journal of Gastroenterology, vol. 23, no. 28, pp. 5097-5114, 2017.

[43] X. Zhong, Y.-J. Surh, S.-G. Do et al., "Baicalein inhibits dextran sulfate sodium-induced mouse colitis," Journal of Cancer Prevention, vol. 24, no. 2, pp. 129-138, 2019.
[44] C.-L. Zhang, S. Zhang, W.-X. He et al., "Baicalin may alleviate inflammatory infiltration in dextran sodium sulfate-induced chronic ulcerative colitis via inhibiting IL-33 expression," Life Sciences, vol. 186, pp. 125-132, 2017.

[45] M.-H. Pan, C.-S. Lai, Y.-J. Wang, and C.-T. Ho, “Acacetin suppressed LPS-induced up-expression of iNOS and COX-2 in murine macrophages and TPA-induced tumor promotion in mice," Biochemical Pharmacology, vol. 72, no. 10, pp. 1293-1303, 2006.

[46] S. Liang, X. Deng, L. Lei et al., "The comparative study of the therapeutic effects and mechanism of baicalin, baicalein, and their combination on ulcerative colitis rat," Frontiers in Pharmacology, vol. 10, p. 1466, 2019.

[47] Y. Zhao, Q. Zhu, X. Bu et al., "Triggering apoptosis by oroxylin A through caspase-8 activation and p62/SQSTM1 proteolysis," Redox Biology, vol. 29, Article ID 101392, 2020.

[48] S. Tm and K. Mk, "Molecular cross-talk between the NRF2/ KEAP1 signaling pathway, autophagy, and apoptosis," Free Radical Biology \& Medicine, vol. 50, no. 9, 2011.

[49] H. Huang, X. Zhang, and J. Li, "Protective effect of oroxylin A against lipopolysaccharide and/or D-galactosamine-induced acute liver injury in mice," Journal of Surgical Research, vol. 195, no. 2, pp. 522-528, 2015.

[50] K. Ding, Y. Y. Tan, Y. Ding et al., " $\beta$-Sitosterol improves experimental colitis in mice with a target against pathogenic bacteria," Journal of Cellular Biochemistry, vol. 120, no. 4, pp. 5687-5694, 2019.

[51] T. Que, Y. Song, Z. Liu et al., "Decreased miRNA-637 is an unfavorable prognosis marker and promotes glioma cell growth, migration and invasion via direct targeting Akt1," Oncogene, vol. 34, no. 38, pp. 4952-4963, 2015.

[52] N. Zhang and X. Chen, "Potential role of O-GlcNAcylation and involvement of PI3K/Akt1 pathway in the expression of oncogenic phenotypes of gastric cancer cellsin vitro," Biotechnology and Applied Biochemistry, vol. 63, no. 6, pp. 841851, 2016.

[53] Y. Liu, X. Liu, W. Hua et al., "Berberine inhibits macrophage M1 polarization via AKT1/SOCS1/NF- $\kappa$ B signaling pathway to protect against DSS-induced colitis," International Immunopharmacology, vol. 57, pp. 121-131, 2018.

[54] T. T. Pizarro, S. A. De La Rue, and F. Cominelli, "Role of interleukin 6 in a murine model of Crohn's ileitis: are cytokine/anticytokine strategies the future for IBD therapies?" Gut, vol. 55, no. 9, pp. 1226-1227, 2006.

[55] Y. S. Kim, Y. Ahn, M. H. Hong et al., "Rosuvastatin suppresses the inflammatory responses through inhibition of c-Jun $\mathrm{N}$-terminal kinase and nuclear factor- $\kappa \mathrm{b}$ in endothelial cells," Journal of Cardiovascular Pharmacology, vol. 49, no. 6, pp. 376-383, 2007.

[56] K. Goh and S.-D. Xiao, "Inflammatory bowel disease: a survey of the epidemiology in Asia," Journal of Digestive Diseases, vol. 10, no. 1, pp. 1-6, 2009.

[57] Y. Ishiguro, "Mucosal proinflammatory cytokine production correlates with endoscopic activity of ulcerative colitis," Journal of Gastroenterology, vol. 34, no. 1, pp. 66-74, 1999.

[58] G. Rogler, "Chronic ulcerative colitis and colorectal cancer," Cancer Letters, vol. 345, no. 2, pp. 235-241, 2014.

[59] E. N. D. Low, N. M. Mokhtar, Z. Wong, and R. A. Raja Ali, "Colonic mucosal transcriptomic changes in patients with long-duration ulcerative colitis revealed colitis-associated cancer pathways," Journal of Crohn's and Colitis, vol. 13, no. 6, pp. 755-763, 2019.

[60] B. Verstockt, G. Van Assche, S. Vermeire, and M. Ferrante, "Biological therapy targeting the IL-23/IL-17 axis in 
inflammatory bowel disease," Expert Opinion on Biological Therapy, vol. 17, no. 1, pp. 31-47, 2017.

[61] A. Geremia and D. P. Jewell, "The IL-23/IL-17 pathway in inflammatory bowel disease," Expert Review of Gastroenterology \& Hepatology, vol. 6, no. 2, pp. 223-237, 2012.

[62] S. Xu and X. Cao, "Interleukin-17 and its expanding biological functions," Cellular \& Molecular Immunology, vol. 7, no. 3, pp. 164-174, 2010.

[63] M. F. Neurath, "IL-23 in inflammatory bowel diseases and colon cancer," Cytokine \& Growth Factor Reviews, vol. 45, pp. 1-8, 2019.

[64] E. Bianchi and L. Rogge, "The IL-23/IL-17 pathway in human chronic inflammatory diseases-new insight from genetics and targeted therapies," Genes \& Immunity, vol. 20, no. 5, pp. 415-425, 2019.

[65] K. Kobayashi, H. Tomita, M. Shimizu et al., "p53 expression as a diagnostic biomarker in ulcerative colitis-associated cancer," International Journal of Molecular Sciences, vol. 18, no. 6, 2017.

[66] H. Su, Q. Kang, H. Wang et al., "Changes in expression of p53 and inflammatory factors in patients with ulcerative colitis," Experimental and Therapeutic Medicine, vol. 17, no. 4, pp. 2451-2456, 2019.

[67] X. L. Huang, J. Xu, X. H. Zhang et al., "PI3K/Akt signaling pathway is involved in the pathogenesis of ulcerative colitis," Inflammation Research, vol. 60, no. 8, pp. 727-734, 2011.

[68] X. Wang, D. Li, Y. Zhang, S. Wu, and F. Tang, "Costus root granules improve ulcerative colitis through regulation of TGF- $\beta$ mediation of the PI3K/AKT signaling pathway," Experimental and Therapeutic Medicine, vol. 15, no. 5, pp. 4477-4484, 2018.

[69] F. Kei, Y. Sho-ichi, and O. Seiya, "Role of AGEs-RAGE system in cardiovascular disease," Current Pharmaceutical Design, vol. 20, no. 14, pp. 2395-2402, 2014.

[70] F. A. Moura, M. O. F. Goulart, S. B. G. Campos, and A. S. da Paz Martins, "The close interplay of nitro-oxidative stress, advanced glycation end products and inflammation in inflammatory bowel diseases," Current Medicinal Chemistry, vol. 27, no. 13, pp. 2059-2076, 2020. 